\title{
Long-term Analgesic Efficacy of An Ultrasound Guided Single-shot Adductor Canal Block in Patients Undergoing Total Knee Arthroplasty
}

\author{
Zhang Y.', Yang J.',Wang X.', Liao R. ', Tan Z.', Liu J.' \\ 'West China Hospital, Sichuan University, Dept of Anaesthesiology, Chengdu, China \\ 2West China Hospital, Sichuan University, Dept of Surgery, Chengdu, China
}

\begin{abstract}
Background and objectives: Both single-shot and continuous adductor canal blocks (ACB) have shown promise in reducing postoperative pain in total knee arthroplasty (TKA) patients. In this prospective, randomized and placebo-controlled study, we compared analgesic efficacy, when given as a single-shot vs. continuous technique with intermittent boluses during the postoperative period.
\end{abstract}

Methods: 75 patients presenting for primary unilateral TKA received preoperatively an ultrasound-guided singleshot $A C B$ and indwelled postoperatively an adductor canal catheter. Those patients were randomly received: a preoperative single-shot of ropivacaine $0.5 \%, 20 \mathrm{ml}$ following intermittent saline boluses at $12 \mathrm{~h}$ and $24 \mathrm{~h}$ postoperatively (S-ACB group), or ropivacaine $0.5 \%, 20 \mathrm{ml}$ preoperatively, $12 \mathrm{~h}$ and $24 \mathrm{~h}$ postoperatively (C-ACB group), or saline preoperatively and postoperatively (control group). The primary outcome was pain with movement on postoperative Dayl and Day2. Other endpoints included static pain scores, quadriceps muscle strength, rescue opioid consumption, cost incurred and patient satisfaction.

Results: Both $A C B$ techniques were equally effective in diminishing dynamic pain and reducing the side effects normally associated with Pethidine Hydrochloride. Dynamic pain scores of the two ACB groups were lower than those of the control group (S-ACB, C-ACB vs. Control group, 4.0, 3.0 vs. 5.0, $p<0.025$ ). Two ACB groups were less likely to use opioids on the first two days after surgery than control (S-ACB vs. C-ACB and control groups, $25.8 \%$, $17 \%$, and $60 \%, P<0.05)$. However, patients receiving a continuous block experienced less quadriceps muscle strength (S-ACB vs. C-ACB group, 4[4,5] vs. 4[3,4], 4[4,5] vs. 4[4,4], respectively, $\mathrm{P}<0.05$ ). Time consumption for a single-shot $A C B$ was 4.0 (I.4) min, which is sharply shorter than those for indwelling an adductor canal catheter (20.0 (5.0) min, $P=0$ ). In addition, there was an extra 80 dollars spent for each adductor canal catheter. Patients receiving a single block experienced more satisfied with their pain-relief treatment (S-ACB vs. C-ACB and control groups, $92 \%$ vs. $74 \%$ and $60 \%, P<0.05)$.

Conclusions: Considering the similar analgesic effect, better quadriceps muscle strength, easier operation and higher patient satisfaction the single-shot ACB might be more suitable for TKA patients. 